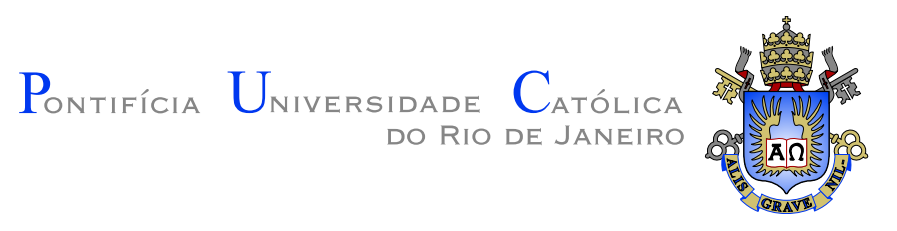

Taís de Sá Pereira

Silhuetas e Linhas Laplacianas de Nuvens de Pontos via Reconstrução Local

Dissertação de Mestrado

Dissertação apresentada como requisito parcial para obtenção do grau de Mestre pelo Programa de Pós-graduação em Matemática do Departamento de Matemática da PUC-Rio.

Orientador: Prof. Sinésio Pesco

Rio de Janeiro setembro de 2013 


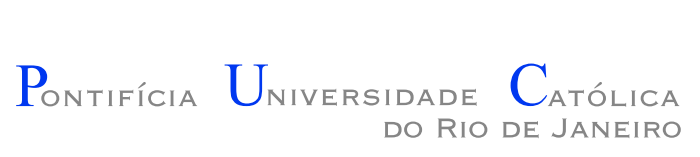

\title{
Silhuetas e Linhas Laplacianas de Nuvens de Pontos via Reconstrução Local
}

\begin{abstract}
Dissertação apresentada como requisito parcial para obtenção do grau de Mestre pelo Programa de Pós-graduação em Matemática do Departamento de Matemática do Centro Técnico Científico da PUC-Rio. Aprovada pela comissão examinadora abaixo assinada.
\end{abstract}

Prof. Sinésio Pesco

Orientador Departamento de Matemática - PUC-Rio

Prof. Dirce Uesu Pesco Instituto de Matemática - UFF

Prof. Hélio Lopes Departamento de Informática - PUC - Rio

Prof. José Eugenio Leal Coordenador do Centro Técnico Científico - PUC-Rio 
Todos os direitos reservados. Proibida a reprodução total ou parcial do trabalho sem autorização da universidade, do autor e do orientador.

\section{Taís de Sá Pereira}

Graduou-se em Licenciatura Plena em Matemática na Universidade Federal Fluminense - UFF.

Ficha Catalográfica

Pereira, Taís

Silhuetas e Linhas Laplacianas de Nuvens de Pontos via Reconstrução Local / Taís de Sá Pereira; orientador: Sinésio Pesco. - Rio de Janeiro : PUC-Rio, Departamento de Matemática, 2013.

v., 54 f: il. ; $29,7 \mathrm{~cm}$

1. Dissertação (Mestrado em Matemática) - Pontifícia Universidade Católica do Rio de Janeiro, Departamento de Matemática.

Inclui referências bibliográficas.

1. Matemática - Tese. 2. Extração de silhueta. 3. Nuvem de pontos. 4. Reconstrução local. 5. Linhas Laplacianas. I. Pesco, Sinésio. II. Pontifícia Universidade Católica do Rio de Janeiro. Departamento de Matemática.

CDD: 510 


\section{Agradecimentos}

Agradeço a Deus pela oportunidade da vida e do estudo.

Aos meus pais Rui, Sonia e Walter, pelo apoio, amor e por terem me ensinado o valor de uma boa educação.

Ao meu orientador, professor Sinesio Pesco, pela paciência, tranquilidade e dedicação para me transmitir seus conhecimentos durante o desenvolvimento do trabalho.

Aos meus amigos da PUC-Rio Pablo, Karen e Rômulo pela companhia nesses últimos anos e, em especial, aos amigos Renata e João, por toda paciência e tempo que dedicaram a me ajudar.

Aos meus amigos e família por compreenderem minha ausência em muitos momentos e pela força que me deram para seguir em frente.

Aos meus alunos por darem mais sentido à minha vida e aos meus colegas de trabalho, em especial aos amigos da E. M. Ceará, pelo apoio durante o curso de mestrado.

Aos membros da comissão examinadora, professora Dirce e professor Hélio, pelas sugestões.

Aos professores e funcionários do departamento de Matemática por toda ajuda.

A CAPES e à PUC-Rio pelos auxílios concedidos. 


\section{Resumo}

Pereira, Taís; Pesco, Sinésio. Silhuetas e Linhas Laplacianas de Nuvens de Pontos via Reconstrução Local. Rio de Janeiro, 2013. 54p. Dissertação de Mestrado — Departamento de Matemática, Pontifícia Universidade Católica do Rio de Janeiro.

No presente trabalho propomos uma nova forma de extrair a silhueta de uma nuvem de pontos, via reconstrução local de uma superfície descrita implicitamente por uma função polinomial. Esta reconstrução é baseada nos métodos Gradient one fitting e Ridge regression. A curva silhueta fica definida implicitamente por um sistema de equações não-lineares e sua geração é feita por continuação numérica. Como resultado, verificamos que nosso método se mostrou adequado para tratar dados com ruídos. Além disso, apresentamos um método para a extração local de linhas laplacianas de uma nuvem de pontos baseado na reconstrução local utilizando a triangulação de Delaunay.

\section{Palavras-chave}

Extração de silhueta; Nuvem de pontos; Reconstrução local; Linhas Laplacianas; 


\section{Abstract}

Pereira, Taís; Pesco, Sinésio (Advisor). Silhouettes and Laplacian Lines of Point Clouds via Local Reconstruction. Rio de Janeiro, 2013. 54p. MSc. Dissertation - Departmento de Matemática, Pontifícia Universidade Católica do Rio de Janeiro.

In this work we propose a new method for silhouette extraction of a point cloud, via local reconstruction of a surface described implicitly by a polynomial function. This reconstruction is based on the Gradient one fitting and Ridge regression methods. The curve silhouette is implicitly defined by a system of nonlinear equations, and is obtained using numerical continuation. As a result, we observe that our method is suitable to handle noisy data. In addition, we present a method for extracting Laplacian Lines of a point cloud based on local reconstruction using the Delaunay triangulation.

\section{Keywords}

Silhouette extraction; Point cloud; Local reconstruction; Laplacian Lines; 


\section{Sumário}

1 Introdução $\quad 10$

1.1 Motivação 10

$\begin{array}{lll}1.2 & \text { Contribuições } & 11\end{array}$

$\begin{array}{lll}1.3 & \text { Trabalhos anteriores } & 11\end{array}$

$\begin{array}{ll}1.4 \text { Divisão da dissertação } & 12\end{array}$

2 Conceitos Básicos $\quad 14$

2.1 Iluminação e Silhueta 14

2.2 Triangulação de Delaunay no plano e Diagrama de Voronoi 15

$\begin{array}{lll}2.3 & \text { Laplaciano } & 17\end{array}$

3 Silhueta de uma nuvem de pontos via reconstrução local de malha $\quad \mathbf{1 9}$

$\begin{array}{lll}3.1 & \text { Reconstrução local de malha } & 19\end{array}$

3.2 Extração local da silhueta de uma nuvem de pontos 22

3.3 Linhas Laplacianas de uma nuvem de pontos 23

$4 \quad$ Silhueta de uma nuvem de pontos via superfícies implícitas $\quad \mathbf{2 7}$

4.1 Reconstrução local das superfícies 27

4.2 Extração local da silhueta de uma nuvem de pontos 31

5 Resultados $\quad 36$

5.1 Extração de silhueta 36

5.2 Silhueta de nuvens com ruídos 44

5.3 Extração de Linhas Laplacianas 45

5.4 Linhas Laplacianas de nuvens de pontos com ruídos 48

6 Conclusão e trabalhos futuros $\quad 52$

$\begin{array}{ll}\text { Referências Bibliográficas } & 53\end{array}$ 


\section{Lista de figuras}

1.1 Netuno: silhueta de uma nuvem de pontos $\quad 10$

1.2 Silhueta de nuvem de pontos com ruídos 12

2.1 Curva silhueta. 14

2.2 Extração da silhueta em cada face de uma malha de triângulos. $\quad 15$

2.3 Exemplos de silhuetas extraídas de malhas poligonais. 15

2.4 Círculo circunscrito não-vazio. $\quad 16$

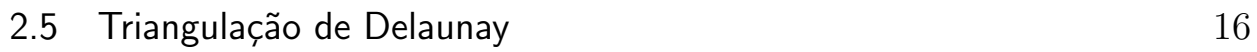

$\begin{array}{lll}2.6 & \text { Peso das cotangentes. } & 18\end{array}$

3.1 Filtro dos pontos vizinhos. 20

3.2 Obtenção da estrela de um ponto 21

3.3 Linhas Laplacianas e silhueta. 24

3.4 Linhas Laplacianas para diferentes valores de $\mathrm{h} \quad 25$

4.1 Interseção de bounding boxes de pontos vizinhos 29

4.2 Método preditor corretor de Euler-Newton. 33

4.3 Silhueta local 34

5.1 Coelho: silhueta via método normal threshold 36

5.2 Coelho: detalhes da silhueta via normal threshold 37

5.3 Coelho: silhueta via reconstrução local de superfície implícita 37

5.4 Coelho: pontos da silhueta via método normal threshold 37

5.5 Cavalo: silhueta sem filtro, $\mathrm{k}=16 \quad 38$

5.6 Cavalo (pata): estrela e silhueta sem filtro, com $k=16 \quad 39$

5.7 Cavalo: silhueta sem filtro, com $k=12$ e $k=7$

5.8 Cavalo: silhueta com filtro $\delta=0.7$, com $\mathrm{k}=12, \mathrm{k}=8$ e $\mathrm{k}=7 \quad 40$

5.9 Mão: silhueta, com $k=8$ e sem filtro 41

5.10 Mão: detalhes entre os dedos, $\mathrm{k}=15$ e $\delta=0.5$

5.11 Coelho: silhueta, com $\mathrm{k}=10$ e filtros $\delta=0.7$ e $\delta=0.5$. 41

5.12 Exemplos de silhueta via reconstrução local de superfícies implícitas 42

5.13 Estratégia 2

5.14 Estratégia 3

5.15 Mão: silhueta da nuvem antes de aplicar a perturbação 44

5.16 Mão: comparação da silhueta de nuvem com ruídos $(p=0.007) \quad 45$

5.17 Mão: comparação da silhueta de nuvem com ruídos $(p=0.013) \quad 45$

5.18 Netuno: comparação da silhueta de nuvem $\operatorname{com} \operatorname{ruídos}(p=0.006) \quad 46$

5.19 Netuno: detalhes da Figura 5.18

5.20 Manequim: comparação da silhueta de nuvem sem ruídos 47

5.21 Manequim: comparação da silhueta de nuvem com ruídos $(p=0.025) 47$

5.22 Laplaciano considerando apenas a estrela do ponto como vizinhança 48

5.23 Dragão: Linhas Laplacianas para diferentes valores de $h \quad 48$

5.24 Linhas Laplacianas de uma nuvem de pontos 49

5.25 Manequim: Linhas Laplacianas e silhueta de nuvem com ruídos $\quad 50$

5.26 Netuno: Linhas Laplacianas e silhueta de nuvem com ruídos 51 